\title{
Original
}

\section{Factores de riesgo asociados a lesiones vesicales en cesárea}

\author{
Jaime Alcocer Urueta, Marcela Bonilla Mares, Viridiana Gorbea Chávez, Beatriz Velázquez Valassi
}

Instituto Nacional de Perinatología. México

\begin{abstract}
Resumen
Objetivo: Identificar los factores de riesgo para lesión vesical durante la operación cesárea.

Material y métodos: Se realizó un estudio de casos y controles de mujeres sometidas a cesárea en el periodo comprendido entre el 1 de enero de 2001 y el 31 de diciembre de 2007 en el INPerIER. Los casos fueron mujeres que habían sufrido lesión vesical en el procedimiento, como controles se seleccionaron al azar 2 mujeres por caso a las que se les realizó cesárea sin lesión vesical en el mismo periodo de tiempo. Se revisaron los expedientes analizando las características demográficas y clínicas las cuales se compararon entre sí.

Resultados: Se encontraron 21 lesiones vesicales entre 24,057 cesáreas (incidencia 0,087\%), de las cuales sólo se analizaron 19. La cesárea previa fue más frecuente en los casos que en los controles (63\% vs 42\% p 0,134), con un Odds Ratio (OR) de 2,35 (IC 95\% 0,759- 7,319), al comparar el antecedente de una cesárea contra ninguna el OR resultó de 3,75 (IC 95\% 1,002- 14,07). Se encontraron diferencias estadísticamente significativas ( $\mathrm{p}<, 05)$ en: edad gestacional $(38,16$ vs 37,35 semanas), una cesárea previa $(42 \%$ vs $18 \%)$, adherencias $(79 \%$ vs $5 \%)$, VBAC $(31,5 \%$ vs $3 \%)$, incisión media ( $16 \%$ vs $68 \%)$, incisión Pfannenstiel $(84 \%$ vs $32 \%)$, hemorragia ( $744 \mathrm{cc}$ vs $509 \mathrm{cc}$ ) y tiempo quirúrgico (135 vs 58 minutos), con lesión vesical y sin ella respectivamente. No se encontraron diferencias significativas en la edad materna, el IMC, cirugías previas, TDP, RPM, altura del feto, corioamnioitis, preinducción, incisión uterina, urgencia del procedimiento o ruptura uterina. La presencia de adherencias tuvo un OR de 67,5 (IC 95\% 11,14- 408).

Conclusiones: $\mathrm{El}$ antecedente de cesárea y de adherencias son factores de riesgo para lesión vesical durante la cesárea.
\end{abstract}

Palabras clave: Lesión vesical. Cesárea. Factores de riesgo.

\section{Risk factors for bladder injuries during cesarean section}

\begin{abstract}
Objective: To identify risk factors for bladder injury during cesarean delivery, to let patients and doctors know them and their importance.

Methods: We conducted a case-control study of women undergoing cesarean delivery at the Instituto Nacional de Perinatologialsidro Espinosa de los Reyes between january 2001 and december 2007. Cases were women with bladder injuries at the time of cesarean section. Two controls per case were selected randomly. Medical records were reviewed for clinical and demographic data to compare them.

Results: Twenty-one bladder injuries were identified among 24, 057 cesarean sections, (incidence $0.087 \%$ ), only 19 were analized. Prior cesarean section was more prevalent among cases than controls (63\% vs $42 \%$ p 0.134), with an OR of 2.35 (95\% CI 0.759 7.319), when we take only patients with one cesarea in contrast with no cesarea the OR is 3.75 (95\% CI 1.002- 14.07). Statistically significant differences (P values < .05) between cases and controls were found in gestacional age (38.16 vs 37.35 weeks), prior cesareans $(42 \%$ vs $18 \%)$, adhesions ( $79 \%$ vs $5 \%)$, Odds ratio of 67.5 (95\% CI 11.14- 408), VBAC (31.5 vs 3\%), median skin incisión (16\% vs $68 \%$ ), Pfannenstiel ( $84 \%$ vs $32 \%$ ), blood loss ( $744 \mathrm{cc}$ vs $509 \mathrm{cc}$ ) and length of surgery 135 vs 58 minutes). No differences were found among age, BMI, prior surgery, labor, premature rupture of membranes, station, chorioamnioitis, induction, uterine incision, timing of delivery, uterine rupture.

Conclusion: Prior cesarean section and adhesions are risk factors for bladder injury at the time of repeat cesarean delivery. Elective cesarean delivery is valid but it is duty of physicians to inform patients the risks of it.
\end{abstract}

Keywords: Bladder injuries. Cesarean section. Risk factors.

A unque poco común, las lesiones vesicales durante la operación cesárea han sido ampliamente reportadas en la literatura con una incidencia de $0,31 \%$. Las series más grandes y referidas en la literatura son las descritas por Eisenkop ${ }^{1,2}$. Esto puede ser controvertido porque con la liberalización de indicaciones para la cesárea, la frecuencia de lesiones vesicales también puede aumentar debido a que su incidencia es mayor que en los partos no $\operatorname{complicados}^{3}$. Hay otros estudios en los que las tasas son mucho menores, refiriendo $0,01 \%$ de lesiones vesicales en todos los nacimientos, siendo de $0,14 \%$ en cesáreas. El diagnóstico de estas es en su mayoría es inmediato y por fortuna la mayor parte se produce en el techo y no afectan al trígono ${ }^{4}$. 
Los factores de riesgo asociados a lesiones de la vías urinarias se pueden clasificar en prequirúrgicos (urgencia del procedimiento, placenta previa, presentación anómala, trabajo de parto distócico, ruptura uterina, cirugías previas, infecciones abdominales o pélvicas previas) e intraquirúrgicos (hemorragia, histerectomía obstétrica, vejiga llena, acretismo, cesárea extraperitoneal) ${ }^{1,5}$. No es raro que después de un trabajo de parto prolongado, sobre todo en nulíparas, se incida en el momento de la cesárea la vagina en lugar del segmento uterino, predisponiendo así a la lesión de la vejiga ${ }^{6}$.

En una serie de casos se vio que los factores de riesgo asociados con lesiones de la vejiga fueron en orden descendente: la formación de colgajo vesical en pacientes con cesáreas previas, en pacientes primigestas, durante la incisión uterina, adherencias a la pared abdominal con cesáreas previas, en cesáreas extraperitoneales por extensiones hacia vejiga o uréter, ruptura uterina, vejiga distendida ${ }^{7}$.

Los factores de riesgo más importantes son las cesáreas de emergencia, en un estudio se vio que hasta el 69\% de las lesiones en cesárea eran en cesáreas de emergencia por baja reserva fetal o desprendimiento previo de placenta normoinserta; otros factores importantes son: cirugías previas, hemorragias obstétricas e histerectomías postcesárea ${ }^{8}$.

En un estudio retrospectivo se observó que la incisión media infraumbilical se asociaba más que la Pfannenstiel con un OR de 6,7 (95\%, IC 2,6 16,5) $\mathrm{P}<0,0001$. Para ambas incisiones las lesiones vesicales aumentaban directamente proporcional al número de cesáreas previas ${ }^{9}$.

Durante el embarazo el aparato genitourinario sufre cambios que ponen a las vías urinarias en una situación de mayor riesgo para que estas se lesionen. Hay una elevación de la vejiga que sigue al crecimiento uterino que sitúa a la vejiga en una posición intrabdominal alta ${ }^{1}$. Una cesárea previa también favorece la adherencia de la vejiga al segmento uterino, haciéndola más propensa a lesiones en el momento de la histerotomía ${ }^{6}$.

Las lesiones vesicales que ocurren durante la operación cesárea son fácilmente detectadas y reparadas. La detección oportuna de las lesiones de las vías urinarias, mientras aún nos encontramos en el quirófano, facilitan una reparación inmediata, generando muy escasa morbilidad. De no ser así pueden haber complicaciones como fístulas o ascitis urinaria que puede llegar a comprometer seriamente a la paciente $^{8,10}$.
El objetivo de nuestro estudio es determinar los factores de riesgo más importantes que intervienen en este tipo de lesiones durante el parto operatorio abdominal.

\section{MATERIAL Y MÉTODOS}

Se realizó un estudio retrospectivo en el que se identificaron los casos de todas las pacientes que tuvieron lesión vesical en cesárea reportadas en la base de datos de la Clínica de Uroginecología del INPerIER en el periodo comprendido entre el 1 de enero de 2001 al 31 de diciembre de 2007, los cuales fueron 21. Se analizaron sólo 19 de estos expedientes, ya que 2 no se encontraron en el archivo, se obtuvieron los datos demográficos y clínicos de estas pacientes. Se utilizaron como controles 38 pacientes a quienes se les realizó cesárea en ese mismo periodo y no tuvieron lesión vesical.

Los datos se recolectaron primero en una hoja de cálculo de Excel, para después ser procesados en el programa SPSS versión 12.

Se identificaron los datos para analizar variables cuantitativas y obtener medidas de tendencia central como promedio y desviación estándar. Se aplicó como prueba estadística la chi cuadrado para variables cualitativas buscando su asociación; y estableciendo de éstas la estimación del riesgo al obtener la razón de momios e intervalos de confianza del 95\%.

\section{RESULTADOS}

En el periodo comprendido entre el 1 de enero de 2001 y el 31 de diciembre de 2007 se realizaron 24,057 cesáreas en el INPerIER. En estos procedimientos se reportaron un total de 21 lesiones vesicales con una tasa de 8,73 por cada 10.000 cesáreas, o bien del 0,08\% (21/24057).

Las características demográficas de los casos y controles se observan en la Tabla 1.

Las características clínicas de los casos y controles se observan en la Tabla 2.

Respecto a las indicaciones de cesárea con y sin lesión vesical, se resumen en la Tabla 3. La indicación más común en los casos fue el antecedente de 2 o más cesáreas y para los controles la cesárea electiva.

Para determinar la asociación entre el riesgo de lesión vesical y otros factores, se realizó el cálculo de la razón de proporción para el Odds ratio e intervalo de confianza del 95\% en cada uno de los casos pertinentes (Tabla 4). La cesárea previa fue más frecuen- 
Tabla 1. Características demográficas de casos y controles.

\begin{tabular}{lccc}
\hline & $\begin{array}{c}\text { Con lesión vesical } \\
\text { Casos n=19 }\end{array}$ & $\begin{array}{c}\text { Sin lesión vesical } \\
\text { Controles n=38 }\end{array}$ & $\boldsymbol{p}$ \\
\hline Edad materna (años) & $32,89 \pm 5,12$ & $33,49 \pm 8,38$ &, 118 \\
IMC (kg/m $\mathrm{m}^{2}$ ) & $29,49 \pm 3,33$ & $30,9 \pm 3,97$ &, 446 \\
Paridad n (\%) & & & \\
$\quad 0$ & $6(31)$ & $8(21)$ &, 384 \\
$\geq 1$ & $13(69)$ & $30(79)$ &, 032 \\
Edad gestacional (SDG) & $38,16 \pm 1,5$ & $37,35 \pm 2,8$ &, 13 \\
Cesáreas previas n (\%) & & &, 05 \\
$\quad 0$ & $7(37)$ & $22(58)$ &, 823 \\
$\quad 1$ & $8(42)$ & $7(18)$ &, 59 \\
$\geq 2$ & $4(21)$ & $9(24)$ & $<, 0001$ \\
Cirugía previa n (\%) & $2(10,5)$ & $6(15,7)$ & $2(5)$ \\
Adherencias n (\%) & $15(79)$ & &
\end{tabular}

Tabla 2. Comparación de características clínicas de casos y controles

\begin{tabular}{lccc}
\hline & $\begin{array}{c}\text { Con lesión vesical } \\
\text { Casos n=19 }\end{array}$ & $\begin{array}{c}\text { Sin lesión vesical } \\
\text { Controles n=38 }\end{array}$ & $\boldsymbol{p}$ \\
\hline TDP n (\%) & $12(63)$ & $21(55)$ &, 569 \\
RPM n (\%) & $6(31,5)$ & $7(18,4)$ &, 264 \\
Encajados n (\%) & $7(36)$ & $11(29)$ &, 546 \\
Corioamnioitis n (\%) & $0(0)$ & $0(0)$ & \\
Preinducción n (\%) & $0(0)$ & $3(8)$ &, 208 \\
Incisión uterina n (\%) & $1(5)$ & $0(0)$ &, 154 \\
$\quad$ Clásica & $18(95)$ & $38(100)$ & \\
$\quad$ Transversa & $8(42)$ & $21(55)$ &, 349 \\
Tipo de urgencia n (\%) & $11(58)$ & $16(42)$ &, 260 \\
$\quad$ Programada & $0(0)$ & $1(3)$ &, 476 \\
$\quad$ Urgente & $6(31.5)$ & $1(3)$ &, 002 \\
$\quad$ Emergente & $1(5)$ & $0(0)$ &, 154 \\
VBAC n (\%) & $3(16)$ & $26(68)$ & $<, 0001$ \\
Ruptura uterina n (\%) & $16(84)$ & $12(32)$ & \\
Incisión en piel n (\%) $\quad$ Media & $744,73 \pm 425,21$ & $509,2 \pm 108,96$ & $<, 0001$ \\
$\quad$ Pfannenstiel & $135,52 \pm 40,16$ & $58,31 \pm 14,99$ & $<, 0001$ \\
\hline Hemorragia (cc) & & & \\
Tiempo quirúrgico (minutos) & & & \\
\hline
\end{tabular}

te en los casos que en los controles (63\% vs $42 \% \mathrm{p}$ 0,134), con OR de 2,35 (IC 95\% 0,759-7,319), al comparar el antecedente de una cesárea contra ninguna el OR resultó de 3,75 (IC 95\% 1,002-14,07).

Todas las lesiones vesicales fueron en el techo con una extensión media de $4,6+3,71 \mathrm{~cm}$ y un rango que iba de $0,5 \mathrm{~cm}$ a $14 \mathrm{~cm}$.

\section{DISCUSIÓN}

Al completar nuestro estudio y analizar los resultados encontramos que estos discrepan con los reportados en la literatura, pero también hallamos algunas coincidencias ya bien definidas.
En nuestro estudió obtuvimos una tasa de lesión vesical de 8,73 por 10.000 cesáreas $(0,08 \%)$, lo que nos deja dentro de los rangos reportados en la literatura que van de $0,0016 \%$ a $0,94 \%$. Las tasas más estándar se reportan en el artículo clásico de Eisenkop de $0,2 \%$ para primíparas y de 0,6\% para pacientes con cesárea previa $^{7}$. Una causa por la cual nuestra tasa es baja pudiera ser la gran cantidad de cesáreas que tenemos, muchas de estas con un riesgo quirúrgico bajo.

Las características de las pacientes en ambos grupos tales como edad, IMC, paridad y presencia de cirugías previas fue muy similar y no se encontraron diferencias significativas. Destacamos en este rubro que la paridad no influyó en la presencia o no de lesión vesical a diferencia de lo reportado en el artículo de Phipps et al. ${ }^{11}$, en el que reporta menos lesiones en primigestas. Otra diferencia respecto a su estudio es que la presencia o no de cirugías previas no impactó sobre el riesgo de lesión. La presencia de adherencias fue un factor muy importante que aumentó el riesgo de lesión 67,5 veces (OR 67,5, IC 95\% 11,14408,79 ), lo cual deja claro la relación entre adherencias y lesión vesical, aunque el intervalo tan amplio se debe a una muestra pequeña $(n=19)$. En cuanto a la incisión en piel, también encontramos discrepancias, si comparamos nuestros resultados donde se presentó más riesgo de lesión en los casos en que se realizó una incisión Pfannenstiel. En el artículo de Makoha et al. ${ }^{9}$ se vio que la incisión media se asociaba con un mayor riesgo que la incisión Pfannenstiel para lesión vesical con un OR crudo de 6,7 (IC 95\%, 2,6-16,5) que después de ajustarse para limpiar los factores confusores (número de cesáreas, experiencia del cirujano y presencia de adherencias), fue de 3,89 (IC $95 \%, 1,4-8,9)$. 
Tabla 3. Indicación de cesárea

\begin{tabular}{|c|c|c|c|}
\hline Indicación de cesárea & $\begin{array}{c}\text { Con lesión vesical=19 } \\
n(\%)\end{array}$ & $\begin{array}{c}\text { Sin lesión vesical }=38 \\
n(\%)\end{array}$ & $\begin{array}{c}\text { Total }=57 \\
\text { n }(\%)\end{array}$ \\
\hline Baja reserva & $1(5,2)$ & $8(21)$ & $9(15,8)$ \\
\hline Condilomatosis & $0(0)$ & $1(2,6)$ & $1(1,7)$ \\
\hline $\mathrm{DCP}$ & $0(0)$ & $2(5,2)$ & $2(3,5)$ \\
\hline Electiva & $1(5,2)$ & $13(34,2)$ & $14(24,5)$ \\
\hline Expulsivo prolongado & $4(21)$ & $0(0)$ & $4(7)$ \\
\hline Falta de progreso de TDP & $1(5,2)$ & $0(0)$ & $1(1,7)$ \\
\hline Gemelar & $0(0)$ & $1(2,6)$ & $1(1,7)$ \\
\hline Iterativa & $6(31,5)$ & $9(23,6)$ & $15(26,3)$ \\
\hline Pélvico & $2(10,5)$ & $3(7,8)$ & $5(8,7)$ \\
\hline Intergenésico corto & $2(10,5)$ & $0(0)$ & $2(3,5)$ \\
\hline Placenta previa & $1(5,2)$ & $0(0)$ & $1(1,7)$ \\
\hline Pretérmino & $0(0)$ & $1(2,6)$ & $1(1,7)$ \\
\hline Riesgo de ruptura uterina & $1(5,2)$ & $0(0)$ & $1(1,7)$ \\
\hline
\end{tabular}

Tabla 4. Factores de riesgo asociados a lesión vesical en cesárea

\begin{tabular}{lcc}
\hline Factor de riesgo & OR & (IC 95\%) \\
\hline Cesárea previa & 2,35 & $(, 759-7.319)$ \\
Una cesárea vs ninguna & 3,75 & $(1,002-14,07)$ \\
Dos cesáreas vs ninguna & 1,39 & $(, 32-5.97)$ \\
Urgente vs programada & 1,69 & $(, 558-5.16)$ \\
VBAC & 17,07 & $(1,87-155,56)$ \\
Adherencias & 67,5 & $(11,14-408,79)$ \\
\hline
\end{tabular}

A diferencia del estudio de Phipps et al. ${ }^{11}$ en donde encontraron aumentado el riesgo de lesión vesical en las pacientes con trabajo de parto, ruptura prematura de membranas, productos encajados, procedimientos urgentes, en nuestro estudio, aunque hubo diferencias similares, no llegaron a ser estadísticamente significativas. En algo que si coincidimos, fue que el intento de parto vaginal después de cesárea aumentaba el riesgo 17 veces para la lesión (OR 17,07, IC 95\% 1,87-155,56). El sangrado y el tiempo quirúrgico mostraron diferencias estadísticamente significativas, siendo mayores en el grupo de lesión vesical en comparación con el grupo control: 744,73 cc $+425,21$ cc vs. 509,2 cc $+108,96$ cc y $135,52 \mathrm{~min}+40,16 \mathrm{~min}$ vs. $58,31 \mathrm{~min}+14,99$ min respectivamente.

La presencia de cesárea previa presentó un OR de 2,35 (IC 95\%, 0,759-7,319), al analizar la presencia de sólo una cesárea previa el OR fue 3,75 (IC 95\%, 1,002-14,07). En este caso la tendencia es clara, pero por diversos factores no resulta comparable con lo reportado en la literatura universal que ya ha dejado bien establecido que la cesárea es un riesgo y que este es directamente proporcional al número de eventos previos.

Así podemos concluir que después de hacer el análisis de la experiencia institucional sobre lesión vesical en cesárea en un periodo de 7 años en un centro con una alta tasa de cesáreas como lo es el nuestro, nos damos cuenta de varios puntos. La tasa de lesión es baja en comparación a los parámetros mundiales y esto pudiera deberse en primer lugar, a un subreporte de lesiones que no fueron diagnosticadas, o por lo menos, consignadas en los expedientes, pero que tampoco produjeron mayor morbilidad a nuestras pacientes. También pudiera deberse como ya se ha dicho en párrafos anteriores a que muchos de nuestros procedimientos son por causas médicas maternas o fetales, las cuales no aumentan la complejidad quirúrgica de los procedimientos. Con esto no queremos decir que esto sea inocuo, ya que está bien establecido el riesgo que implica la cesárea y más a mayor número de estas, no sólo como riesgo para lesión vesical, lo cual encontramos en nuestro estudio y en toda la literatura que fue revisada, sino también como riesgo para placenta previa y/o acretismo que pudieran terminar en histerectomía obstétrica y aumentar el riesgo de hemorragia, la cual hoy por hoy sigue siendo la primera causa de muerte materna. Es por eso importante tratar de disminuir el índice de cesáreas con la idea en mente de que las recomendaciones de la Norma Oficial Mexicana son de $20 \%$ para centros de concentración y de $15 \%$ para hospitales de segundo nivel. El ACOG también tiene como meta 
para el 2010 un índice de 15\% de cesáreas en primigestas y de $37,5 \%$ para pacientes con cesárea previa.

En la literatura, así como en nuestro estudio, resultó muy importante el hecho de la presencia de adherencias, lo que aumenta la posibilidad de lesión vesical, esto pudiera controlarse tratando de disminuir las intervenciones pélvico-abdominales innecesarias, tomando en cuenta la posibilidad de cirugias de mínima invasión o en los casos que sea necesario acceder a la cavidad, hacerlo sin dañar los tejidos, corroborando la hemostasia y tratando de no introducir objetos extraños que pudieran aumentar la aparición de dichas adherencias. El pronóstico de la lesión vesical después de un diagnóstico oportuno con una correcta reparación es muy bueno y prácticamente no deja secuelas, sobre todo si la lesión no involucra la zona del trígono ${ }^{12}$.

Podemos finalizar con lo importante que es tener siempre presente el riesgo latente de la lesión vesical, conocer los factores asociados, particularizar los casos y siempre proceder con una técnica quirúrgica meticulosa para así poder disminuir la morbilidad en nuestras pacientes. Una técnica cuidadosa y metódica siempre es recomendable, sobre todo en casos difíciles donde el riesgo de lesión aumenta.

\section{REFERENCIAS}

1. Lucci JA. Lesiones Urológicas y Gastrointestinales. En: Gilstrap LC, Cunningham FG, Van Dorsten JP. Urgencias en salas de partos y obstetricia quirúrgica; $2^{\mathrm{a}}$ Edición. Madrid: Panamericana; 2004. pp. 443-452.
2. Buchholz NP, Grandeau E, Huber-Buchholz M. Urological complications associated with cesarean section. Eur J Obstet Gynecol Reprod Biol. 1994; 56(3):161-163.

3. Faricy PO, Auspurger RR, Kaufman JM. Bladder injuries associated with cesarean section. J Urol. 1978;120(6):762-763.

4. Rajasekar D, Hall M. Urinary tract injuries during obsteric intervention. Br J Obstet Gynaecol. 1997;104(6):731-734.

5. Miklos JR, Sze E, Parobeck D, Karram MM. Vesicouterine fistula: a rare complication of birth after cesearean. Obstet Gynecol. 1996;86(4 Pt 2):638-639.

6. Davis JD. Management of injuries to the urinary and gastrointestinal tract during cesarean section. Obstet Gynecol Clin North Am. 1999;26(3):469-481.

7. Eisenkop CM, Richman R, Platt LD, Paul R. Urinary tract injury during cesarean section. Obstet Gynecol. 1982;60(5): 591-597.

8. Yossepowitch O, Baniel J, Livne PM. Urological injuries during cesarean section: intraoperative diagnosis and management. J Urol. 2004;173(3):1049.

9. Makoha FW, Fathuddien MA, Felimban HM. Choice of abdominal incision and risk of trauma to de urinary bladder and bowel in multiple cesarean sections. Eur J Obstet Gynecol Reprod Biol. 2006;125(1):50-53.

10. Williams CH, Heaney JA, Young W. Respiratory distress following cesarean section: cryptic presentation of bladder injury. Urology. 1994;44(3):441-443.

11. Phipps MG, Watabe B, Clemons JL, Weitzen S, Myers DL. Risk factors for bladder injury during cesarean delivery. Obstet Gynecol. 2005;105(1):156-160.

12. Onuora VC, Ariyan AL, Koko AH, Wahab A, Al Jawini N. Major injuries to de urinary tract in association with childbirth. East African Medical Journal. 1997;74(8):523-528.

Correspondencia autor: Dr. Jaime Alcocer Urueta

Instituto Nacional de Perinatología. México

Montes Urales 800 Colonia Lomas Virreyes - 11000 Delegación

Miguel Hidalgo Distrito Federal, México

Tel.: 55209900

E-mail autor: jaime_alcocer@yahoo.com.mx

Información artículo: Original - Incontinencia

Trabajo recibido: septiembre 2008

Trabajo aceptado: abril 2009 